\title{
The Use of Descriptives and Expressives by ESL Learners: Investigating the Relevance of Interpretation and Context.
}

\author{
Hamza Alshenqeeti* \\ Department of Languages and Translation, School of Arts and Humanities, Taibah University, Madinah, Saudi \\ Arabia
}

*Corresponding Author: Hamza Alshenqeeti, Department of Languages and Translation, School of Arts and Humanities, Taibah University, Madinah, Saudi Arabia.

\begin{abstract}
Expressives and descriptives are units of language which serve to reinforce meaning and intent within an utterance. These linguistic devices include taboo words, exclamations and honorifics. Much of the work undertaken in the area has been focused on identifying the different functions that these units of language perform within speech and written work. There are also indications that context may be a factor in how they are received by listeners. Less attention has however been given to how English as a Second Language (ESL) learners may interpret expressives and descriptives and what influence and impact context has on whether the literal or implied meaning is taken from the sentence. Seventy-five ESL learners, living and working in the United Kingdom evaluated ten sentences, with and without context for their meaning and expressive use. The results suggest that context is highly significant, as is social and cultural understanding. This has implications for applied linguistics research, ESL teaching and learning, suggesting that achievement of native-like speech, which includes effective and appropriate use of expressives and descriptives needs to not only include context but also cross-cultural training and understanding of social norms in the target language. It is however recognised that additional research into expressives and descriptives in an ESL context is necessary.
\end{abstract}

Keywords: Context, Descriptives, ESL, Expressives, Interpretation, and Native-like speech.

\section{INTRODUCTION}

\subsection{Background to the Study}

Expressives are an element of language which serves to enhance and strengthen the intent of a message (Jay and Janschewitz, 2007). This can be in the form of honorifics, exclamations or polite terms which are generally used when the speaker wishes to emphasise a particular aspect of their speech. This is to convey the strength of the speaker's emotion (exclamations), to express respect for the listener (honorifics) or an emphasis without resorting to invasive language (polite terms) (c.f.Mildenberger, 2017). However, the way that these elements of language are used may potentially be scenario and context-dependent in regard to how they are both delivered and received and thus evaluated (Croom, 2011). For ESL learners, interpreting the context and thus the intended role of the expressive or descriptive can be challenging (Aftab, 2017). In addition, descriptives, which assign a quality to a subject "blue" or "large" for instance, are utilised to avoid restrictions in modification of expressions and can also be challenging, if not subject to confusion, misinterpretation or misuse by language users such as EFL learners. Furthermore, these two elements of language are integral to the delivery of interesting, well-rounded and native-like speech. Therefore, understanding how they are interpreted and the impact of context on their use and understanding is an area which is worthy of deeper exploration.

\subsection{Aim of the Study}

The aim of this work is to examine in greater detail the use of expressives and descriptives in the oral production of L2 speakers. In particular, it aims to identify when and how EFL learners feel that different types of expressives should be applied to reinforce an utterance, alongside their use of descriptives and quality assignment of terms to emphasise meaning. Specifically, given that English can be a highly colourful language in terms of both expressives and descriptives, whether there are 
The Use of Descriptives and Expressives by ESL Learners: Investigating the Relevance of Interpretation and Context.

contextual or cultural variations in the way that learners of English apply their knowledge of when and how these devices should be used is hence investigated throughout this paper.

\subsection{Questions of the Study}

The questions to be answered in order to achieve the aim of the current study were as followed:

- What understanding do advanced ESL learners have of the use of expressives in oral production?

- Is there a significant variation based on context in how extensively ESL learners use expressives and descriptives during L2 oral production?

- Is there a cultural impact from cultural factors in which expressives are used by speakers of English?

- Are there recommendations that can be made to facilitate teaching of these linguistic devices to speakers of English?

\subsection{Rationale for the Study}

As ESL learners increase their proficiency, their aim is to be as native-like in their speech and expressions as possible. Recognising this, the use and knowledge of expressives and how they can reinforce and emphasise meaning is a crucial skill to acquire. Furthermore, effective use of descriptives demonstrates a wider knowledge and more creative approach to utterance generation than using simple terms when giving information or engaging in verbal interaction with native English speakers. Therefore, understanding whether there are barriers to the effective use of these linguistic devices based on context and interpretation by ESL learners is both a timely and relevant work to undertake.

\subsection{Structure of the Paper}

The remainder of the paper is divided into four sections. Following this introduction, a review of existing viewpoints in the area of ESL usage of expressives and descriptives is undertaken. This will identify key terms and perspectives in the area. Following this, a brief outline of a primary data exercise methodology is provided, and a fourth section detailing the results of these and how they align with existing viewpoints. Finally, a conclusion drawing, and overall evaluation of the area is provided, along with recommendations for applied linguistics and English language teaching practices and any further areas of investigation.

\section{ReVieW OF EXISTING Perspectives}

\subsection{Introduction}

According to Potts (2005),expressives should be viewed as a form of conventional implicature, for instance in the sentence "that blasted dog is growling again" the implication is that the dog is not liked. They may be used in a number of ways which are explored later in this section but essentially serve to convey to the listener an indication of their views on the subject being discussed. A difficulty here is that descriptives have been suggested as being part of this implicative class, and therefore words may be viewed in terms of expression (Geurts, 2007), but also in terms of descriptives and it is here potentially that the challenge of interpretation and context may arise for ESL learners who are not necessarily familiar with English cultural norms in the usage of expressives despite indications from Vingerhoets et al.(2013) that these emotive turns may be a biological imperative for conveying strong emotion. The particular challenge is identifying from interpretation of the actual words, combined with context whether the word is being used in an expressive or descriptive manner, or whether the function is a combination of both. This section examines existing works and perspectives in the area in order to identify the differences between expressives and descriptives when used by ESL learners, but also to provide a foundation for examination of primary data.

\subsection{Expressives and Descriptives in Speech}

Expressives is the term given to morphemes, words and grammatical constructions that convey emotion and feeling, as identified by Cruse (1986) and Potts (2005). This includes words such as "idiot", and other epithets, but also adjectives that take an attributive role, such as "damn". Expressives also includes honorifics, occasionally suffixes, but at the same time also play a descriptive role in delivering colourful, creative language that emphasises or changes the meaning of 
an utterance from either speaker or listener perspective (Harris and Potts, 2009). In grammatical terms, expressives may be literal such as in "the main road" or "the bloody clothes" (indicating clothes covered in blood) where the meaning is exact and not open to interpretation (Potts et al, 2009). However, the use of expressives has a strong impact on context, and are often used negatively or destructively. In effect, the use of expressives is a clue to the perspective of the speaker and thus potentially the focus and outcomes of any interaction that follows their use (Kratzer, 2004). Moreover, expressive terms can also be interpreted in a variety of ways, depending on the relationship between interlocutors and the context of the utterance. As a result, their use can lead to either misunderstanding, conflict or miscommunication, even when the intent was to reinforce a view and provide more weight to the utterances being made. This means that there needs to be understanding of the role that expressives, and indeed descriptives take in speech acts.

\subsection{The Role of Expressives and Descriptives in Speech}

Importantly, expressives and indeed their counterpart descriptives are a powerful tool for reinforcing a message, and achieving a strong reaction or conveying the emotional state of the speaker (Traugott, 1982; Potts, 2007). Despite their strong role in providing cues to emotion, and intent, understanding the reliability of expressive signals could be challenging for ESL learners, and therefore understanding how to define these may be crucial for effective teaching approaches. However, the area is not very well explored, and focus has generally been on the use of these elements of language, or in expressive writing, rather than identifying whether context plays a part in interpretation (Smith, 2013; Mercury, 1995; Horan, 2013).In this context, as Liyanage et al, (2015) suggest, explicit instruction in expressives, particularly those that are focused on swearing or taboo language would be beneficial to ESL students wishing to achieve a native like speech in English. However, in order to be able to do this, greater understanding of the role of expressives and descriptives as emotive terms is required.

In this regard, Potts (2007 and 2005) indicates that there are a number of roles that expressives may taken within an utterance, and in relation to the descriptive function of the word, as shown in the figure below:

Figure 1. Types of Expressives (Source: Potts, 2007)

\begin{tabular}{|l|l|}
\hline \multicolumn{1}{|c|}{ Expressive Type } & \multicolumn{1}{c|}{ Function } \\
\hline Independence & Acts separately from the descriptive content to give an additional layer of meaning \\
\hline Non-displaceability & Acts to give meaning to an element of the situation in which the utterance is made \\
\hline Perspective Dependence & $\begin{array}{l}\text { The expressive word used is evaluated from the perspective of the speaker but may } \\
\text { also be from the perspective of the listener }\end{array}$ \\
\hline Descriptive Ineffability & An extension and thus building on of an expressive content via descriptive terms \\
\hline Immediacy & $\begin{array}{l}\text { Act as a performative so that the aim of using an expressive is achieved through } \\
\text { speaking it, without offering content }\end{array}$ \\
\hline Repeatability & Where an expressive is used more than once to strengthen emotive content \\
\hline
\end{tabular}

Reviewing the various types in Figure 1, and re-evaluating whether expressives are truly conversational implicatures, it can be seen that descriptive ineffability, immediacy and repeatability are not conventional implicatures, but the first three are (Potts, 2007b). What this can lead to is a question over whether the use of the expressive to give a description or to meet one of the requirements of the speaker indicated in the table above.

Epithets are a particular form of expressive that can cause difficulties. Whilst these are part of the overall morphosyntactic system, using examples from other languages there appears to be evidence for expressives to be seen as a linguistically coherent class within the overall grammar system. In this respect, Aoun and Choueiri (2000),Aounet al.(2001), and Sells and Kim (2006) note that Japanese and Arabic use of epithets leads to specific and predictable morphosyntactic changes. What this suggests is that despite the various functions of expressives, there may be linguistic coherence of the words, based on a clear distinction at the semantic level between expressive and descriptive content (Potts, 2007). However, this distinction may only be identified when there is a context for the expression also provided the construction of a phrase.

An extensive evaluation of this perspective can be found in Potts et al.(2008) who suggest that descriptives and expressives act differently to satisfy identity conditions across a range of languages. 
However, they also suggest, in line with Pullum and Rawlins (2007), that these conditions are focused on descriptive semantic content and that expressives may act differently. This again highlights the potential difficulties that ESL learners may face when dealing with expressives and descriptives and underlines the need for investigation of the area with primary data. To explore how best to achieve an understanding of the impact of context on interpretation of expressives and descriptives, the following section provides a summary of the approaches adopted to gather relevant data.

\section{MeThodology}

\subsection{Introduction}

The approach to gathering data for the evaluation of the impact of interpretation and context on the use of expressives and descriptives was based on the framework indicated by Saunders et al.(2011) for effective study design. In this framework, the first area of consideration is the focus and approach of the work. Given the limited examination in the field of the impact of context and interpretation on the use of expressives and descriptives, it was felt that an exploratory approach was required (Churchill and Brown, 2007). The rationale underlying this choice is that an exploratory focus is best applied when the focus of a work is to achieve understanding of a subject area or a phenomenon, rather than hard scientific fact, cause and effect relationships or answers to "why" questions (Baines and Chansarker, 2002; Saunders et al, 2011). Furthermore, as McNabb (2004) notes, when there is a high potential for new questions or avenues of research to emerge, it is best to follow an exploratory route. Combined with this approach, it was elected to adopt the philosophy of direct realism, viewing the outcomes and evaluations based on observations in a real-world context (Blaikie, 2009). This is important because as Constant et al.(2009) notes, expressive and descriptives research has been limited by the constraints of laboratory settings, or a focus on wide corpora rather than individual perspectives, particularly those of ESL learners. Complementing this realism underpinning was an inductive perspective based on the development of theories via observation and collection of data, rather than assessment and testing of a previously-defined hypothesis (Blaikie, 2009).

\subsection{Collection of Data and Participant Sample}

The strategy for gathering data to evaluate expressives and descriptives was to engage advanced learners of English and ask them to participate in a short questionnaire. The aim was to provide them with sentences using both expressives and descriptives, with a context and ask them to identify, from a multiple-choice selection the implication of the meaning behind use of the expressive or descriptive term. The rationale for this approach was that it would provide indications of how the students interpreted the usage of the linguistic devices and thus provide answers to the study questions and achieve the aim of the work. The sampling was purposive in nature, as the students were selected based on their proficiency levels of English, with the criteria being that they were living and working or studying in the UK and had advanced English skills.

\subsection{Survey Instrument Design}

To allow for a wider sample to be surveyed, a quantitative approach was adopted for the questionnaire. The instrument had two parts, the first focused on gathering basic demographic data such as age, gender and time spent in the UK, as well as nationality. This was due to the view that this could be relevant impacting variables on the responses. The second part was focused on the sentences using descriptives and expressives with the responses being in the form of a multiple-choice selection. The sentences themselves come from adaptations of those used Potts (2007) and Constant et al, (2009) and their studies on semantic and inferential context in the use and understanding of expressives and descriptives. The aim was to use sentences that could be determined as demonstrating potential ambiguity in terms of meaning depending on context, and these two studies both work to clarify this potential ambiguity. By giving the respondents the sentences without context first, and then a context, it was intended to evaluate how the addition of context changed interpretation. In this way, statistical outcomes could be identified to support analysis of trends and patterns in the interpretation of expressives and the impact of context by the participants. 


\subsection{Analysis of Raw Data}

The analysis of the raw questionnaire data was achieved through inputting into SPSS statistical software. The rationale for using this approach is that the software allows for evaluation and analysis of correlations, frequencies and thus patterns within the numerical data to emerge. At the same time, it allows for the undertaking of validity and reliability measures as well as internal consistency of the data which indicates how well any findings may be generalised outside of the sample group.

\subsection{Ethical Considerations}

Babbie and Benaquisto (2009) indicate that any study which is based in real world observation and participants must ensure adherence to strict ethical guidelines in regard to ensuring informed consent, confidentiality of responses and clear indications of what is required. To achieve this, the questionnaire was prefaced with a brief statement indicating the purpose of the questionnaire, what participation would require and an indication that confidentiality and anonymity would be maintained at all times.

\subsection{Validity and reliability}

The term "reliability" in relation to examination of a study area refers to the level of dependability of any data collected. In effect, whether similar outcomes would be achieved in a different research setting and thus whether the work has meaning and relevance. For this investigation, reliability was achieved by ensuring there was a clear reflection of the research aims in the way that the questions were framed and analysed. In a similar vein, the term "validity" refers to the effectiveness of the actual process of data collection, and whether it is measuring the right elements and furthermore whether the analysis undertaken reflects reality. For this work, the statistical analysis of learner responses to situational use of expressives and descriptives was felt to meet this criteria for validity, following indications by Saunders et al.(2011).

\subsection{Limitations}

With a recognition that the data was not collected in a highly controlled, laboratory setting so that real world responses would be achieved, there is also understanding of limitations to this process. These include a relatively small sample, which means if the study was undertaken with a larger group, and a different cohort make-up, alternative outcomes could result. In addition, using only one data collection approach (i.e. quantitative) meant that reasons behind selections could not be evaluated in greater depth. However, it was felt that sufficient indications could be achieved through the processes adopted to ensure an effective outcomes and meeting of the objectives.

\subsection{Summary}

The incorporation of expressives and descriptives in the oral production of ESL speakers provides a means of delivering more creative, colourful utterances. However, there is a potential that their usage and thus implication may be misunderstood based on context or interpretation by learners. The approaches adopted to evaluate these views were selected to provide a baseline understanding of where challenges and barriers may exist based on understanding of the terms and their usage, but also potentially cultural factors in interpretation and social norms. The outcomes of the questionnaire analysis and how they contribute to existing works are provided in the following section.

\section{Data ANALYSiS}

\subsection{Introduction}

A total of 75 individuals completed the full questionnaire. A further 15 only partially completed the instrument so were discounted from the final analyses. Each of the sentences was evaluated for the role of the expressive and the meanings assigned with and without context. In addition, crossevaluation was undertaken to identify whether cultural background, age or gender had an impact on the responses given. In terms of the cohort make-up there was however a bias to males, who made up $64 \%$ of the group. The reason for this was due to the contacts made for the sampling, but given the small size it was felt this would not unduly impact on the overall findings. In regard to age, as the figure below indicates the majority were aged over 30, and none were over 35 . Despite this bias to younger ESL speaker, it was felt again that this did not necessarily detract from the overall findings. 
The Use of Descriptives and Expressives by ESL Learners: Investigating the Relevance of Interpretation and Context.

Figure 2. Age of Participants

\begin{tabular}{|l|l|l|l|l|l|}
\hline \multicolumn{2}{|c|}{ Age } \\
\hline \multirow{3}{*}{ Valid } & \multicolumn{1}{|c|}{ Frequency } & \multicolumn{1}{c|}{ Percent } & Valid Percent & Cumulative Percent \\
\cline { 2 - 6 } & $18-25$ & 13 & 17.3 & 17.3 & 17.3 \\
\cline { 2 - 6 } & $26-30$ & 23 & 30.7 & 30.7 & 48.0 \\
\cline { 2 - 6 } & $31-35$ & 39 & 52.0 & 52.0 & 100.0 \\
\cline { 2 - 6 } & Total & 75 & 100.0 & 100.0 & \\
\hline
\end{tabular}

Ethnicity too showed some bias to Arabic ESL learners, as the figure on the next page indicates but as there was representation from other cultures, it was felt that some initial perspectives on the impact of culture could be achieved with this group.

Figure 3. Ethnicity of Group

\begin{tabular}{|c|c|c|c|c|c|}
\hline \multicolumn{6}{|c|}{ Ethnicity } \\
\hline & & Frequency & Percent & Valid Percent & Cumulative Percent \\
\hline \multirow[t]{4}{*}{ Valid } & Arabic & 38 & 50.7 & 50.7 & 50.7 \\
\hline & Asian & 21 & 28.0 & 28.0 & 78.7 \\
\hline & Eurasian & 16 & 21.3 & 21.3 & 100.0 \\
\hline & Total & 75 & 100.0 & 100.0 & \\
\hline
\end{tabular}

Having indicated the general make-up of the respondents, the specific questions were assessed for the interpreted meaning with and without context.

\subsection{Interpretation with and without Context}

The first sentence "that villain James is late again", when considered with and without context, showed general similarity in understanding. However, $4 \%$ took the meaning literally, as shown in the figure below. In general, however, the implied meaning that James was late, and "villain" was acting as an independence expressive, giving additional meaning of annoyance was identified by the majority.

\begin{tabular}{|l|l|l|}
\hline \multicolumn{2}{|c|}{ "That villain James was late again" } \\
\hline \multicolumn{1}{|c|}{ Without Context } & With Context \\
\hline The speaker does not like James & 29.3 & 29.3 \\
\hline The speaker believes James to be a bad person & 26.7 & 18.7 \\
\hline The speaker believes James to be illegitimate & 4.0 & \\
\hline The speaker is annoyed with James & 40.0 & 52.0 \\
\hline
\end{tabular}

Those that took the literal meaning were either Arabic or Asian, and none of the Eurasian respondents considered this to be the intent of the sentence. In sentence two, however, "That rascal Kresge is so famous", there was distinct variation when context was added as the figure below indicates.

Figure 4. Sentence 2

\begin{tabular}{|c|c|c|}
\hline \multicolumn{3}{|l|}{ "That Rascal Kresge is So Famous." } \\
\hline & Without context & With Context \\
\hline The speaker views Kresge as famous & 16.0 & \\
\hline The speaker does not like Kresge & 16.0 & 46.7 \\
\hline The speaker believes Kresge is a bad person & 4.0 & 2.7 \\
\hline $\begin{array}{l}\text { The speaker is looking for agreement from the listener that Kresge's fame } \\
\text { is undeserved }\end{array}$ & 64.0 & 50.7 \\
\hline
\end{tabular}

Whilst the use of the word rascal, in this context can be viewed as taking a perspective independence function (Potts, 2007), the indications of negativity from the speaker about the subject are reinforced when context is added, at least for these participants. This suggests that context can have a major influence on how the use of an expressive is interpreted. There was no significant influence from ethnicity, age or gender for these outcomes.

For statement 3 however, "That book was fishmonger brilliant", which can be deemed as a descriptive ineffability, i.e. There is an emphasis created by the use of "fishmonger" on how brilliant the speaker thought the word was. When context was added, there was a variation in terms of the content identifying that the book was good, or encouraging the listener to read it. 
The Use of Descriptives and Expressives by ESL Learners: Investigating the Relevance of Interpretation and Context.

Figure 5. Sentence 3

\begin{tabular}{|c|c|c|}
\hline \multicolumn{3}{|c|}{ "That book was fishmonger brilliant" } \\
\hline & Without Context & With Context \\
\hline The speaker is emphasising how good they thought the book was & 61.3 & 48.0 \\
\hline The speaker is trying to shock the audience using swear words & 12.0 & 8.0 \\
\hline The speaker is trying to encourage the listener to read the book & 26.7 & 44.0 \\
\hline
\end{tabular}

Whilst the use of the word rascal, in this context can be viewed as taking a perspective independence function (Potts, 2007), the indications of negativity from the speaker about the subject are reinforced when context is added, at least for these participants. This suggests that context can have a major influence on how the use of an expressive is interpreted. There was no significant influence from ethnicity, age or gender for these outcomes.

For statement 3 however, "That book was fishmonger brilliant", which can be deemed as a descriptive ineffability, i.e. There is an emphasis created by the use of "fishmonger" on how brilliant the speaker thought the word was. When context was added, there was a variation in terms of the content identifying that the book was good, or encouraging the listener to read it.

Figure 6. Sentence 4

\begin{tabular}{|c|c|c|}
\hline \multicolumn{3}{|l|}{ Here's to you, yascoundrel" } \\
\hline & Without Context & Percent \\
\hline The speaker is very fond of the listener and villain is a term of endearment & 48.0 & 44.0 \\
\hline The speaker does not like the subject of the sentence & 1.3 & \\
\hline The speaker is referring to the paternity of the subject & 1.3 & \\
\hline The speaker is celebrating the subject & 49.3 & 56.0 \\
\hline
\end{tabular}

Both with and without context, the sentence was interpreted as being positive and the use of the expressives was a form of immediacy and indications of positivity about the subject. For this sentence therefore, the interpretation appeared to be clear to the participants in terms of offering an indication of the speaker's intent and emotional feelings. This concurs with the work of Potts and Schwartz (2008) and their indications that expressives used to convey emotion do not always require context.

In terms of expressives acting as a performative, this was illustrated in Sentence 5, and indeed when context was provided there was some clear variation as the figure on the next page indicates.

Figure 7, Sentence 5

\begin{tabular}{|l|l|l|}
\hline \multicolumn{2}{|c|}{ "I promise that I will wash the dishes later." } \\
\hline
\end{tabular}

Without context, the sentence was interpreted as predominantly an indication that at present there was no intent to do the dishes immediately. However, when the context of a teenager speaking to a parent was added, this interpretation was diminished in favour of defining an obligation, confirming the performative expressive function. As Schlenker (2007) suggests, when expressives act as performatives, they deliver an obligation from the speaker to the listener. However, without the context, the respondents had varying perspectives on the intent of the speaker. This indicates again the potential that there is an influence from context on interpretation of meaning when an expressive or descriptive is used as Lambe and McLeod (2005) note.

The influence was context was also seen in Sentence 6, which shows repeatability and thus the heightened emotional state of the speaker.

Figure 8. Sentence 6

\begin{tabular}{|l|l|l|}
\hline \multicolumn{3}{|c|}{ "Damn, I left my damn wallet in the car." } \\
\hline & Without Context & With Context \\
\hline The speaker is frustrated by the act of leaving their wallet behind & 34.7 & 48.0 \\
\hline The speaker is being sarcastic and does not wish to pay for something & 4.0 & 4.0 \\
\hline The speaker wishes the listener to pay for something & 61.3 & 48.0 \\
\hline
\end{tabular}


The Use of Descriptives and Expressives by ESL Learners: Investigating the Relevance of Interpretation and Context.

When context was added, i.e. that of a husband and wife, there was a reduction in the view that there was an underlying meaning that the listener should pay, and a greater focus on the speaker's frustration than when there was no context. The reasons behind this are not clear but suggest that context may provide a greater level of information for pragmatic evaluation of the inclusion of an expressive in an utterance in line with the views of Constant et al, (2009).

This was very clear in Sentence 7 as the figure below highlights.

Figure 9. Sentence 7

\begin{tabular}{|l|l|l|}
\hline \multicolumn{2}{|c|}{ "You're such an idiot!" } \\
\hline & \multicolumn{1}{|c|}{ Without Context } & With Context \\
\hline Speaker thinks listener is foolish & 46.7 & \\
\hline Speaker is annoyed with listener & 45.3 & 53.3 \\
\hline Speaker is frustrated by something & 8.0 & 46.7 \\
\hline
\end{tabular}

Without the added context of a husband and wife undertaking DIY, the majority of the respondents interpreted this sentence literally. However, this view was altered with the context, removing the opinion to a more immediate view that the speaker was annoyed, or frustrated. This sentence thus illustrates the perspective dependence function of expressives, as the sentence could be interpreted by either speaker or listener. Clearly for the respondents this was the case with some viewing it as frustration, and some as annoyance. In this regard as Potts (2007) notes, context is crucial in determining the intended and received meaning.

For Sentence 8 there was a similar level of variation, indicating again perspective dependence ambiguity as the figure below indicates:

Figure 10. Sentence 8

\begin{tabular}{|l|l|l|}
\hline \multicolumn{3}{|c|}{ "Please, I am like, so totally over him, I can't begin to tell you!" } \\
\hline \multicolumn{1}{|c|}{ Without Context } & With Context \\
\hline The speaker is highly emotional about the situation & 41.3 & 24.0 \\
\hline The speaker wants to reinforce their feelings on the situation & 8.0 & 2.7 \\
\hline The speaker wants to talk about the situation & 50.7 & 73.3 \\
\hline
\end{tabular}

In this case without context, the interpretation was at an emotional level. However, when the context of two teenage girls was added, the interpretation was moved to a desire to speak about the situation. In this respect, the speaker can be viewed as applying the dependence ineffability of expressive use, using descriptive terms to build on the meaning (Blakemore, 2011). At the same time, the context highlights a potentially more accurate interpretation of intent. One of the difficulties with expressives and particularly their interpretation by ESL learners is that there may be more than one way of determining the intent of the speaker. This is the case in Sentence 9, as shown below.

Figure 12. Sentence 9

\begin{tabular}{|c|c|c|}
\hline \multicolumn{3}{|l|}{ "Best bloody film ever" } \\
\hline & Without Context & With Context \\
\hline The speaker is excited and exuberant about the movie & 46.7 & \\
\hline The speaker is inviting debate and argument about the film & 53.3 & 46.7 \\
\hline The speaker is demonstrating their strong emotions about the film & & 53.3 \\
\hline
\end{tabular}

Before being given the context, the respondents viewed the utterance as being an indication of the speaker's view, and thus there was a descriptive focus to the expressive use. However, once context was provided, this view was altered and there was indication of strong emotional responses and a desire to discuss the film that would not be present in the indications of exuberance identified without context. Aguertet al. (2010) identified contextual bias in interpretation of expressives in children, and this may also be the case for ESL learners. Without context, the interpretation and meaning may be misjudged and thus be subject to errors in responses. The influence of context would therefore appear to be relevant in interpretation of expressives and descriptives. Indeed, there did appear to be some contextual and interpretative misunderstanding in the final sentence, as shown below. 
The Use of Descriptives and Expressives by ESL Learners: Investigating the Relevance of Interpretation and Context.

Figure 13. Sentence 10

\begin{tabular}{|c|c|c|}
\hline \multicolumn{3}{|c|}{ "This place is ridiculously cool" } \\
\hline & Without Context & With Context \\
\hline The speaker believes the place is cold & 33.3 & \\
\hline The speaker is expressing a positive view of the place & 14.7 & 37.3 \\
\hline The speaker is expressing a negative view of the place & 6.7 & \\
\hline The speaker believes the place is stupid & 45.3 & 62.7 \\
\hline
\end{tabular}

As with sentence one, there was an adoption of literal meaning when no context was provided. In addition, the indications even with context that the place was "stupid", presumably based on the use of the word "ridiculous" in a descriptive way. In addition, the change in meaning for a positive view suggests that for some of the cohort there was a recognition of the social function of "ridiculously cool" for indicating a positive view. However, for the majority this was not the case. What this last question highlights is that not only is context important, but also a level of socio-cultural knowledge for EFL learners when interpreting expressives and descriptives use. In this regard as Aydemir et al, (2014) the teaching of English, including effective and appropriate use of expressives needs to incorporate cross-cultural awareness. This is also in line with the work of Mercury (1995) and Horan (2013) regarding the value of learning how taboo language and expressives are used in the target culture for increasing native like speech and interpretation by ESL learners.

\subsection{Summary}

The evaluation of the respondents' interpretation of 10 sentence which employ expressives has indicated that context does appear to have an influence on understood meaning. In addition, there may be variation in this influence depending on the role that the expressive takes within the utterance. The final section thus considers these factors and what implications this may have in terms of ESL teaching in relation to expressives and descriptives.

\section{Discussion AND EVAluation}

The focus of this paper was on identifying whether context had a significant impact on how learners of English as a Second Language (ESL) interpreted the use of expressives and descriptives. These elements of language have been indicated by Potts (2007) to be emotive terms which can either reinforce an utterance, or indicate the feelings of the speaker. At the same time, they can also perform an obligatory act, reinforce meaning or be used as part of social norms. In isolation, their usage can be difficult for ESL learners to judge and thus acquire accurate understanding of how to use them.

\subsection{Evaluation and Implications}

Using 75 ESL learners with advanced proficiency, working or studying in the UK, it was identified that the views of Potts (2007), Constant et al.(2009) and others regarding the importance of pragmatic and contextual information in interpreting expressives has relevance for ESL learners. In particular, theuse of expressives as anti-honorifics and perspective dependence factors led to variation in the interpretations. Whilst the work of Potts and Constant has been focused on English language in general, rather than the ESL context, there is still application in an ESL context. The indications of a lack of knowledge about the social-cultural usage of some of the expressives utilised in this work underlines the need for cultural familiarity to be incorporated into ESL teaching. A surprising find was that there was no major cultural variation in the way that individuals interpreted the sentences, nor was there an impact from age or gender. However, larger samples may achieve different results as would a sample which included students with variable proficiency levels.

\subsection{Concluding Remarks}

The major implication of this work, and the evaluation of expressives and descriptives is that any teaching approaches would need to ensure that contextual information is provided along with the terms so that students understand the role of expressives in a real-life environment. As these devices allow utterances to reinforce emotions, actions and intent it is important that students understand not only how to use them, but crucially how to interpret them. Given the indications of the influence of context on how these units of grammar are interpreted, there is a clear value in introducing both taboo words, and other expressive formats into the ESL classroom. This would enable students to develop 
more expressive, creative natural speech but also enhance their interactions with native speakers outside the classroom. Expressives are a language unit which reinforces meaning and intent, and therefore being able to effectively interpret a speaker's underlying meaning is both a vital skill and a contributor to creative, meaningful language. At the same time, the functions of these units, along with the descriptives to which they are closely aligned, can vary depending on context and thus interpretation. As an initial evaluation of how this applies in an ESL context therefore, this work has highlighted that there is further exploration required into the area at a deeper level and with larger samples.

\section{REFERENCES}

[1] Aftab, A.(2017). Chapter Three Principled Application of Authenticity as An Alternative Teaching Paradigm for PakistaniESL Classrooms. Authenticity in Materials Development for Language Learning, P.44.

[2] Aguert, M., Laval, V., Le Bigot, L. and Bernicot, J.(2010). Understanding expressive speech acts: the role of prosody and situational context in French-speaking 5-to 9-year-olds. Journal of speech, language, and hearing research, 53(6), pp.1629-1641.

[3] Aoun, J. and Choueiri, L.(2000). Epithets. Natural Language \& Linguistic Theory, 18(1), pp.1-39.

[4] Aoun, J., Choueiri, L. and Hornstein, N.(2001). Resumption, movement, and derivational economy. linguistic Inquiry, 32(3), pp.371-403.

[5] Aydemir, E. and Mede, E.(2014). Integrating Target Culture in EFL Classrooms. Turkish Online Journal of Qualitative Inquiry, 5(1), pp.20-33.

[6] Babbie, E.R. And Benaquisto, L., (2009). Fundamentals of Social Research. Cengage Learning.

[7] Baines, P. and Chansarkar, B., (2002), Introducing Marketing research, John Wiley \& Sons Inc, London, UK.

[8] Blaikie, N., (2009), Designing Social Research, New York: Polity Press

[9] Blakemore, D., (2011). On the descriptive ineffability of expressive meaning. Journal of Pragmatics, 43(14), pp.3537-3550.

[10] Churchill, G. A. Jr, and Brown, J. T. (2007), Basic Marketing Research, $5^{\text {th }}$ edition, Thomson, London, UK

[11] Constant, N., Davis, C., Potts, C. and Schwarz, F., (2009). The pragmatics of expressive content: Evidence from large corpora. Sprache und Datenverarbeitung, 33(1-2), pp.5-21.

[12] Croom, A.M., (2011). Slurs. Language Sciences, 33(3), pp.343-358.

[13] Cruse, D.A., (1986). Lexical semantics. Cambridge: Cambridge University Press.

[14] Geurts, B. (2007). Really f-ing brilliant. (Commentary on Christopher Potts, The expressive

[15] dimension.). Theoretical Linguistics 33:209-214.

[16] Harris, J.A. and Potts, C., (2009). Perspective-shifting with appositives and expressives. Linguistics and Philosophy, 32(6), pp.523-552.

[17] Horan, G., (2013). 'You taught me language; and my profit on't/Is, I know how to curse': cursing and swearing in foreign language learning. Language and Intercultural Communication, 13(3), pp.283-297.

[18] Jay, T. and Janschewitz, K., (2007). Filling the emotion gap in linguistic theory: Commentary on Potts' expressive dimension. Theoretical Linguistics, 33(2), pp.215-221.

[19] Kratzer, A., (2004). Interpreting focus: Presupposed or expressive meanings. Theoretical Linguistics, 30(1), pp.123-136.

[20] Lambe, J.L. and McLeod, D.M., (2005). Understanding Third-Person Perception Processes: Predicting Perceived Impact on Self and Others for Multiple Expressive Contexts. Journal of Communication, 55(2), pp.277-291.

[21] Lasersohn, Peter. (2005). Context dependence, disagreement, and predicates of personal taste. Linguistics and Philosophy 28:643-686.

[22] Liyanage, I., Walker, T., Bartlett, B. and Guo, X., (2015). Accommodating taboo language in English language teaching: Issues of appropriacy and authenticity. Language, Culture and Curriculum, 28(2), pp.113-125.

[23] McNabb, D.E., (2004), Research Methods for Political Science, New York: ME Sharpe

[24] Mercury, R.E., (1995). Swearing: A" bad" part of language; A good part of language learning. TESL Canada journal, 13(1), pp.28-36. 
The Use of Descriptives and Expressives by ESL Learners: Investigating the Relevance of Interpretation and Context.

[25] Mildenberger, C.D.(2017). Expressives, Majoratives, and Ineffability. Kriterion., [Online], available from http://www.kriterion-journal-of-philosophy.org/kriterion/issues/Permanent/Kriterion-mildenberger-01.pdf, [accessed 5.12.2017].

[26] Potts, C. (2005). The Logic of Conventional Implicatures: Oxford Studies in TheoreticalLinguistics. Oxford: Oxford University Press.

[27] Potts, C. (2007). Expressive content and the semantics of contexts NSF Grant No. BCS-0642752., [Online], available from http://web.stanford.edu/ cgpfPototts/expressives/docs/potts-nsf-expressivesdescription.pdf, [accessed 4.12.2017].

[28] Potts, C.(2007b) Conventional implicatures, a distinguished class of meanings. The Oxford handbook of linguistic interfaces, pp.475-501.

[29] Potts, C., Asudeh, A., Cable, S., Hara, Y., McCready, E., Alonso-Ovalle, L., Bhatt, R., Davis, C., Kratzer, A., Roeper, T. and Walkow, M., (2009). Expressives and identity conditions. Linguistic Inquiry, 40(2), pp.356-366.

[30] Potts, C., and Schwarz, F., (2008), Exclamatives and Heightened Emotion: Extracting Pragmatic Generalisations from Large Corpora, Ms., UMass Amherst, pp.1-29.

[31] Pullum, G.K. and Rawlins, K., (2007). Argument or no argument? Linguistics and Philosophy, 30(2), pp.277-287.

[32] Saunders, M., Lewis, P. and Thornhill, A. (2011), Research Methods for Business Students, 4th edition, Prentice Hall, London, UK.

[33] Schlenker, P., (2007). Expressive presuppositions. Theoretical Linguistics, 33(2), pp.237-245.

[34] Sells, P. and Kim, J.B., (2006). Honorification in Korean as expressive meaning. Korean Linguistics, 13(1), pp.167-195.

[35] Smith, C., (2013). Creative writing as an important tool in second language acquisition and practice. The Journal of Literature in Language Teaching, 2(2), pp.11-18.

[36] Traugott, E.C., (1982). From propositional to textual and expressive meanings: Some semantic-pragmatic aspects of grammaticalization. Perspectives on historical linguistics, pp.245-271.

[37] Vingerhoets, A.J., Bylsma, L.M. and De Vlam, C., (2013). Swearing: A biopsychosocial perspective. Psihologijske teme, 22(2), pp.287-304.

\section{AUTHOR's BIOGRAPHY}

Hamza Alshenqeeti is an Assistant Professor of Applied Linguistics at Taibah University in Saudi Arabia. He holds a PhD in Applied Linguistics from Newcastle University and an MA in Applied Linguistics from Essex University, England. His research interests revolve around classroom discourse, sociolinguistics, CALL, and language teacher training.

Citation: Hamza Alshenqeeti "The Use of Descriptives and Expressives by ESL Learners: Investigating the Relevance of Interpretation and Context.." International Journal on Studies in English Language and Literature (IJSELL), vol 5, no. 11, 2017, pp. 33-43. doi:http://dx.doi.org/10.20431/2347-3134.0511003.

Copyright: (C) 2017 Authors. This is an open-access article distributed under the terms of the Creative Commons Attribution License, which permits unrestricted use, distribution, and reproduction in any medium, provided the original author and source are credited. 